\title{
Zeno and anti-Zeno effects in two-level systems
}

\author{
Alfredo Luis* \\ Departamento de Óptica, Facultad de Ciencias Físicas, Universidad Complutense, 28040 Madrid, Spain
}

(Received 2 February 2003; published 30 June 2003)

\begin{abstract}
We examine the appearance of Zeno and anti-Zeno effects when the unitary evolution of a two-level system is observed. We demonstrate that, contrary to previous claims, the anti-Zeno effect can occur in two-level systems so that the observation can enhance the transition probability. Moreover, for nonresonant couplings we show that the Zeno and anti-Zeno effects always appear sequentially in the evolution of the same system: there is Zeno effect in the short-time regime and anti-Zeno effect in the long-time regime.
\end{abstract}

DOI: 10.1103/PhysRevA.67.062113

PACS number(s): 03.65.Xp, 42.50.- $\mathrm{p}, 32.80 .-\mathrm{t}$

\section{INTRODUCTION}

One of the appealing consequences of quantum mechanics is that the observation unavoidably disturbs the observed system. This is particularly revealed by the so-called Zeno and anti-Zeno effects [1-5]. The Zeno (anti-Zeno) effect refers to the inhibition (acceleration) of the evolution when attempts are made to observe it. They can be regarded as two particular consequences of the disturbance of the observed system caused by quantum observation.

In this paper, we study the appearance of these effects in the most simple example of quantum physics: a two-level system experiencing a purely unitary evolution causing transitions between the two levels. On the one hand, the existence of the Zeno effect in this system is well known [1,2,69]. On the other hand, it has been claimed that the anti-Zeno effect cannot take place in two-level systems $[2,10]$.

It is worth noting that most approaches to the problem focus on the case of exact resonance. In this work, we remove this restriction considering also nonresonant systems. It is known that the lack of resonance hinders level transitions. The idea is that the quantum observation might block the detuning so that the measurement would stimulate the transition (anti-Zeno effect) [3].

Following this reasoning, we will demonstrate that there can be the anti-Zeno effect in two-level systems. Moreover, we will show that for nonresonant systems, the Zeno and anti-Zeno effects always take place sequentially occupying two different time scales of the evolution of the system.

Unfortunately, the terminology regarding the anti-Zeno effect may be rather confusing, especially because in the literature we can find two terms ("anti-Zeno" and "inverse Zeno") both referring to two different phenomena.

The phenomenon addressed in this work is the acceleration of the original dynamics of the system caused by observation. We use the term "anti-Zeno effect," which is the terminology adopted in most works on the subject $[3,4]$. Nevertheless, other authors use the term "inverse Zeno effect" to refer to this same phenomenon $[2,5,10]$.

The confusion increases when we notice that other authors use these same terms to refer to an entirely different phenomenon also caused by measurement. This is the com-

\footnotetext{
*Electronic address: alluis@ fis.ucm.es
}

plete removal of the original dynamics of the system and its replacement by an externally controlled evolution along a prescribed trajectory [11]. This is referred to as the anti-Zeno effect by some authors [12] and as inverse Zeno effect by some other authors [13].

\section{UNOBSERVED EVOLUTION}

Let us consider a two-dimensional Hilbert space spanned by the orthogonal states $|0\rangle,|1\rangle$. The most general unitary evolution is governed by a Hamiltonian of the form

$$
H=\hbar \Delta|0\rangle\langle 0|+\hbar \frac{\Omega}{2}(|0\rangle\langle 1|+| 1\rangle\langle 0|),
$$

where $\Delta$ is the detuning or mismatch and $\Omega$ is the coupling constant (the Rabi frequency) of the term stimulating the level transitions (see Fig. 1). The most paradigmatic example of this situation is provided by a two-level atom interacting with a classical monochromatic electromagnetic wave. In such a case, $\Delta=\omega-\omega_{0}$, where $\omega$ is the frequency of the field and $\omega_{0}$ is the resonant frequency of the atom.

If at $t=0$ the system is in the state $|1\rangle$, the probability that the system continues in the initial state at later times is

$$
P(t)=1-\frac{\Omega^{2}}{\Omega^{2}+\Delta^{2}} \sin ^{2}\left(\sqrt{\Omega^{2}+\Delta^{2}} t / 2\right) .
$$

Since, in general, this is a rapidly oscillating function, it can be helpful to compute the mean occupation of the initial state

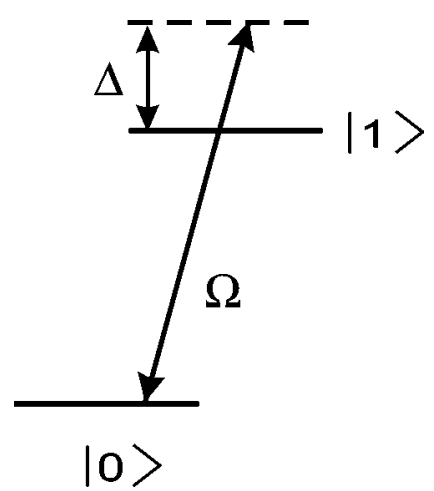

FIG. 1. Scheme of a two-level system. 
$\bar{P}$ as the time average of $P(t)$ over a time interval $T$ long enough such that $\sqrt{\Omega^{2}+\Delta^{2}} T \gg 1$,

$$
\bar{P}=\frac{1}{T} \int_{t-T / 2}^{t+T / 2} d t^{\prime} P\left(t^{\prime}\right) \simeq 1-\frac{1}{2} \frac{\Omega^{2}}{\Omega^{2}+\Delta^{2}} .
$$

It can be appreciated that the mismatch $\Delta$ impedes the transition $|1\rangle \rightarrow|0\rangle$. In particular, when $\Delta \gg \Omega$ we have $P(t)$ $\simeq \bar{P} \simeq 1$ and the system is always close to the initial state.

This situation suggests that there might be room for the anti-Zeno effect according to the following scenario: the quantum observation might block the effect of the detuning favoring in this way the global efficiency of the transition process. A related precedent can be found in Ref. [3]. Therefore, we will focus mainly on strongly nonresonant systems $\bar{P} \simeq 1$ unless otherwise stated. We will study whether the observation of the evolution can stimulate the transition $|1\rangle$ $\rightarrow|0\rangle$. To this end, we will consider discrete as well as continuous observations.

\section{DISCRETE MEASUREMENTS}

For the sake of simplicity, we can begin with the most simple case of ideal, instantaneous, and fully accurate observations of the level occupied $(|0\rangle$ or $|1\rangle)$ at some definite time instants $t=n \tau$, where $\tau$ is the time interval of free evolution between consecutive measurements, and $n$ $=0,1, \ldots$. In these conditions, and assuming the standard state reduction, the probability $p(n)$ that all the first $n$ measurements confirm that the system remains in the initial state is

$$
p(n)=[P(\tau)]^{n} .
$$

For short enough $\tau$, i.e., $\sqrt{\Omega^{2}+\Delta^{2}} \tau \ll 1$, we have

$$
p(n) \simeq e^{-n \Omega^{2} \tau^{2} / 4}=e^{-\Omega^{2} t \tau / 4},
$$

which is independent of $\Delta$. Incidentally, this confirms the above guess concerning the removal of the mismatch caused by the observation.

It is clear from Eq. (5) that if the measurement is frequent enough (i.e., $\tau \rightarrow 0$ ), the evolution of the system will be halted and the observed system will tend to be always in the initial state. This is the Zeno effect. Since for practical reasons $\tau$ is always finite, the Zeno effect will take place provided that the evolution time $t$ is not too large (we refer to this condition as the short-time regime). For example, from Eq. (5) we get a necessary condition $t \ll 1 /\left(\Omega^{2} \tau\right)$ as an upper bound for the duration of the short-time regime.

On the other hand, if we observe the evolution during a period of time exceeding the short-time condition [i.e., the long-time regime $\left.t \gg 1 /\left(\Omega^{2} \tau\right)\right]$, we have that $p(n) \simeq 0$ and the transition $|1\rangle \rightarrow|0\rangle$ will take place with certainty. This is the germ of the anti-Zeno effect (enhancement of the transition probability caused by observation). Next we develop and make more precise this idea.
To this end, we can compute the average probability $\mathcal{P}(t)$ that the observed system is in the initial state $|1\rangle$ at time $t$ $=n \tau$ irrespective of the results of the preceding measurements. Following Ref. [2], we have

$$
\mathcal{P}(t)=\frac{1}{2}\left[1+R_{3}(n \tau)\right],
$$

where $R_{3}$ is the third component of the Bloch vector. If we discard the outcome, we have that each measurement projects the Bloch vector to the third axis $\left(R_{1} \rightarrow 0, R_{2} \rightarrow 0\right.$, $R_{3} \rightarrow R_{3}$ ). From the free evolution between measurements, we have

$$
R_{3}(n \tau)=\mathcal{U}(\tau) R_{3}[(n-1) \tau]=[\mathcal{U}(\tau)]^{n},
$$

where

$$
\mathcal{U}(\tau)=1-\frac{2 \Omega^{2}}{\Omega^{2}+\Delta^{2}} \sin ^{2}\left(\sqrt{\Omega^{2}+\Delta^{2}} \tau / 2\right) .
$$

For frequent enough measurements $\sqrt{\Omega^{2}+\Delta^{2}} \tau \ll 1$, we obtain

$$
\mathcal{P}(t) \simeq \frac{1}{2}\left(1+e^{-\Omega^{2} t \tau / 2}\right) .
$$

Therefore, in the long-time regime $t \gg 1 /\left(\Omega^{2} \tau\right)$, we get $\mathcal{P}(t) \simeq 1 / 2$.

This demonstrates that there can be the anti-Zeno effect in unitarily evolving two-level systems. When $\Delta \neq 0$, the observed occupation of the initial state can be lesser than the unobserved one, $\mathcal{P}(t)<\bar{P}$, for $t$ in the long-time regime. This is particularly clear in the limit of large mismatch $\Delta \gg \Omega$, since in such a case when $t \rightarrow \infty$ we have $\mathcal{P} \simeq 1 / 2$ while $P$ $\simeq \bar{P} \simeq 1$. In other words, the observation stimulates the transition $|1\rangle \rightarrow|0\rangle$.

On the other hand, for perfect resonance we have only the possibility of the Zeno effect: $\Delta=0$ implies $\bar{P}=1 / 2$ so that $\mathcal{P}(t) \geqslant \bar{P}$ for every $t$. This was the situation assumed in Refs. $[2,10]$. Nevertheless, we will show below that for continuous observation there is the possibility of the anti-Zeno effect even for exact resonance.

From the above analysis, we can derive a further interesting result: when $\Delta \neq 0$, the Zeno and anti-Zeno effects are always present sequentially in the evolution of the same system. The two time regimes are separated by the instant of time $t_{0}$ for which the observed probability equals the average unobserved one, $\mathcal{P}\left(t_{0}\right)=\bar{P}$,

$$
t_{0}=\frac{2}{\Omega^{2} \tau} \ln \left(1+\frac{\Omega^{2}}{\Delta^{2}}\right) .
$$

This time instant separates the Zeno regime $[\mathcal{P}(t)>\bar{P}$ for $t$ $\left.\ll t_{0}\right]$ from the anti-Zeno regime $\left[\mathcal{P}(t)<\bar{P}\right.$ for $\left.t \gg t_{0}\right]$. 


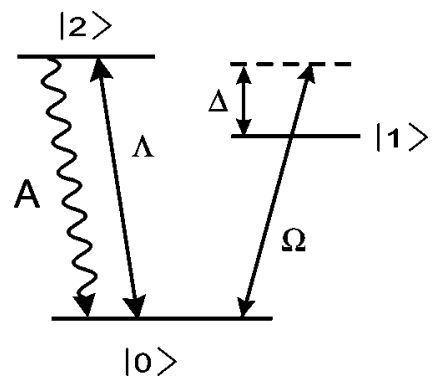

FIG. 2. Scheme of the measurement of the populations of the two-level system $|0\rangle,|1\rangle$.

\section{CONTINUOUS OBSERVATION}

Next we examine the case of continuous observation, i.e., the original evolution and the measurement coexist during the whole process. A very useful practical scheme is sketched in Fig. 2. The state $|0\rangle$ is resonantly coupled to a third auxiliary level $|2\rangle$ (Rabi frequency $\Lambda$ ) that decays spontaneously back to $|0\rangle$ with the emission of photons at a rate $A$. The presence or absence of the emitted photons reveals that the observed system is in the state $|0\rangle$ or $|1\rangle$, respectively $[7,8,14]$.

The joint evolution of the apparatus-system ensemble has been well analyzed in Ref. [15] including the possibility of detuning $\Delta \neq 0$. For example, it has been shown that in the regime $\Lambda, A \gg \Omega$, the occupation of the level $|1\rangle$ can be described by the rate equation [15]

$$
\dot{\mathcal{P}}=-\left(R_{+}+R_{-}\right) \mathcal{P}+R_{+},
$$

leading to

$$
\mathcal{P}(t)=\frac{R_{-}}{R_{+}+R_{-}} e^{-\left(R_{+}+R_{-}\right) t}+\frac{R_{+}}{R_{+}+R_{-}},
$$

where $R_{ \pm}$are the upward and downward transition rates depending on the parameters $\Lambda, A, \Omega, \Delta$. For example, when $A \gg \Lambda \gg \Delta$ we have $R_{+} \simeq R_{-}$[15] so that $\mathcal{P}(t) \simeq 1 / 2$ when $t$ $\rightarrow \infty$. This agrees well with the results of the ideal discrete measurement analyzed above. This is consistent since when
$A \gg \Lambda$, this scheme becomes a faithful implementation of an ideal projective measurement performed at a rate $\tau$ $\simeq 4 A / \Lambda^{2}[6,8,16]$.

We can examine a slightly different regime in which the existence of anti-Zeno is further demonstrated. When $\Lambda$ $\gg \Delta \gg A$, the downward transitions are more likely than the upward ones, $R_{-} \gg R_{+}[9,15]$, so that $\mathcal{P}(t) \simeq 0$ when $t \rightarrow \infty$ and the observed system tends to abandon completely the initial state. This conclusion can be extended to the exact resonance. When $\Delta=0$ and $\Lambda \gg A$ we get again $R_{-} \gg R_{+}$ [15], so that also on resonance there is the anti-Zeno effect in the long-time regime: $\mathcal{P}(t) \simeq 0$ versus $\bar{P}=1 / 2$.

This last measuring condition $\Lambda \gg A$ differs from the schemes analyzed above $A \gg \Lambda$ in that for $\Lambda \gg A$ there is no ideal state reduction, in the sense that the emission of photons in the transition $|0\rangle \rightarrow|2\rangle$ does not reduce the system to the state $|0\rangle$. Instead, the system rapidly reaches a $50 \%$ incoherent superposition of $|0\rangle$ and $|2\rangle$ [17]. Despite this, we stress that the measurement is always fully accurate concerning the correct inference of the system state (no photons if $|1\rangle$ and photons if $|0\rangle$ ). In other words, this is not an example of the partial Zeno effect studied in Ref. [18].

\section{CONCLUSIONS}

In conclusion, we have examined the possibility of the Zeno and anti-Zeno effects in the most simple quantum system: a unitarily evolving two-level system. We have shown that, contrary to previous claims, the anti-Zeno effect can occur, especially in the nonresonant case. For nonresonant systems, the Zeno and anti-Zeno effects appear always sequentially in the evolution of the same system: Zeno effect in the short-time regime and anti-Zeno effect in the long-time regime. These results are relevant given the simplicity and importance of two-level systems, both from the theoretical and experimental points of view. Moreover, this is an interesting example of selective tailoring of the evolution caused by quantum measurement: the observation blocks the effects of detuning, while favors level transitions. Other examples of dynamics constrained or tailored by quantum observation can be found in Ref. [19].
[1] A. Peres, Am. J. Phys. 48, 931 (1980); C. Presilla, R. Onofrio, and U. Tambini, Ann. Phys. (N.Y.) 248, 95 (1996); D. Home and M.A.B. Whitaker, ibid. 258, 237 (1997).

[2] P. Facchi and S. Pascazio, Progress in Optics, edited by E. Wolf (Elsevier, Amsterdam, 2001), Vol. 42, p. 147.

[3] A. Luis and L.L. Sánchez-Soto, Phys. Rev. A 57, 781 (1998); A. Luis, ibid. 66, 012101 (2002).

[4] B. Kaulakys and V. Gontis, Phys. Rev. A 56, 1131 (1997); L. Mišta, Jr., J. Herec, V. Jelínek, J. Řeháček, and J. Peřina, J. Opt. B: Quantum Semiclassical Opt. 2, 726 (2000); A.G. Kofman and G. Kurizki, Nature (London) 405, 546 (2000); O.V. Prezhdo, Phys. Rev. Lett. 85, 4413 (2000); M. Lewenstein and K. Rzążewski, Phys. Rev. A 61, 022105 (2000); A. Marchewka and Z. Schuss, ibid. 61, 052107 (2000); J. Řeháček, J. Peřna,
P. Facchi, S. Pascazio, and L. Mišta, Jr., ibid. 62, 013804 (2000); B. Elattari, and S.A. Gurvitz, ibid. 62, 032102 (2000); A.G. Kofman, G. Kurizki, and T. Opatrný, ibid. 63, 042108 (2001); S.W. Kim, Y.-T. Chough, and K. An, ibid. 63, 052104 (2001); J. Ruseckas and B. Kaulakys, ibid. 63, 062103 (2001); I. Antoniou, E. Karpov, G. Pronko, and E. Yarevsky, ibid. 63, 062110 (2001); M.C. Fischer, B. Gutiérrez-Medina, and M.G. Raizen, Phys. Rev. Lett. 87, 040402 (2001); J. Lawrence, J. Opt. B: Quantum Semiclassical Opt. 4, S446 (2002); K. Koshino, Phys. Rev. A 67, 042101 (2003).

[5] P. Facchi and S. Pascazio, Phys. Rev. A 62, 023804 (2000); P. Facchi, H. Nakazato, and S. Pascazio, Phys. Rev. Lett. 86, 2699 (2001).

[6] W.L. Power and P.L. Knight, Phys. Rev. A 53, 1052 (1996); A. 
Beige and G.C. Hegerfeldt, J. Phys. A 30, 1323 (1997); Chr. Balzer, R. Huesmann, W. Neuhauser, and P.E. Toschek, Opt. Commun. 180, 115 (2000); P.E. Toschek and Chr. Wunderlich, Eur. Phys. J. D 14, 387 (2001); P.E. Toschek and Chr. Balzer, J. Opt. B: Quantum Semiclassical Opt. 4, S450 (2002); Chr. Balzer, Th. Hannemann, D. Reiß, Chr. Wunderlich, W. Neuhauser, and P.E. Toschek, Opt. Commun. 211, 235 (2002).

[7] S. Slijkhuis, G. Nienhuis, and R. Morgenstern, Phys. Rev. A 33, 3977 (1986); W.M. Itano, D.J. Heinzen, J.J. Bollinger, and D.J. Wineland, ibid. 41, 2295 (1990); V. Frerichs and A. Schenzle, ibid. 44, 1962 (1991); E. Block and P.R. Berman, ibid. 44, 1466 (1991); M.J. Gagen and G.J. Milburn, ibid. 47, 1467 (1993); P. Kwiat, H. Weinfurter, T. Herzog, and A. Zeilinger, Phys. Rev. Lett. 74, 4763 (1995); P. Kwiat, H. Weinfurter, T. Herzog, A. Zeilinger, and M. Kasevich, Ann. N.Y. Acad. Sci. 755, 383 (1995); B. Nagels, L.J.F. Hermans, and P.L. Chapovsky, Phys. Rev. Lett. 79, 3097 (1997); K. Mølhave and M. Drewsen, Phys. Lett. A 268, 45 (2000); T. Nakanishi, K. Yamane, and M. Kitano, Phys. Rev. A 65, 013404 (2002); P. Valente, H. Failache, and A. Lezama, ibid. 65, 023814 (2002).

[8] A. Beige and G.C. Hegerfeldt, Phys. Rev. A 53, 53 (1996); L.S. Schulman, ibid. 57, 1509 (1998).

[9] F.B. Jong, R.J.C. Spreeuw, and H.B. van Linden van den Heuvell, Phys. Rev. A 55, 3918 (1997).

[10] A.D. Panov, Phys. Lett. A 298, 295 (2002).

[11] Y. Aharonov and M. Vardi, Phys. Rev. D 21, 2235 (1980); H.F.
Hofmann, G. Mahler, and O. Hess, Phys. Rev. A 57, 4877 (1998).

[12] A.P. Balachandran and S.M. Roy, Phys. Rev. Lett. 84, 4019 (2000).

[13] T.P. Altenmüller and A. Schenzle, Phys. Rev. A 48, 70 (1993); M. Kitano, ibid. 56, 1138 (1997); M. Kitano, K. Yamane, and T. Ikushima, ibid. 59, 3710 (1999).

[14] W. Nagourney, J. Sandberg, and H. Dehmelt, Phys. Rev. Lett. 56, 2797 (1986); Th. Sauter, W. Neuhauser, R. Blatt, and P.E. Toschek, ibid. 57, 1696 (1986); J.C. Bergquist, R.G. Hulet, W.M. Itano, and D.J. Wineland, ibid. 57, 1699 (1986).

[15] H.J. Kimble, R.J. Cook, and A.L. Wells, Phys. Rev. A 34, 3190 (1986).

[16] C. Cohen-Tannoudji and J. Dalibard, Europhys. Lett. 1, 441 (1986); A. Schenzle and R.G. Brewer, Phys. Rev. A 34, 3127 (1986); P. Zoller, M. Marte, and D.F. Walls, ibid. 35, 198 (1987); M. Porrati and S. Putterman, ibid. 36, 929 (1987).

[17] D.T. Pegg and P.L. Knight, Phys. Rev. A 37, 4303 (1988).

[18] A. Peres and A. Ron, Phys. Rev. A 42, 5720 (1990); T.F. Jordan, E.C.G. Sudarshan, and P. Valanju, ibid. 44, 3340 (1991).

[19] A. Luis and L.L. Sánchez-Soto, Phys. Rev. A 60, 56 (1999); K. Machida, H. Nakazato, S. Pascazio, H. Rauch, and S. Yu, ibid. 60, 3448 (1999); P. Facchi, V. Gorini, G. Marmo, S. Pascazio, and E.C.G. Sudarshan, Phys. Lett. A 275, 12 (2000); P. Facchi, S. Pascazio, A. Scardicchio, and L.S. Schulman, e-print quant-ph/0101037; A. Luis, Phys. Rev. A 63, 052112 (2001); J. Opt. B: Quantum Semiclassical Opt. 3, 238 (2001). 